\title{
Investigation of pausing fermentation of salamis with multispectral imaging for optimal
} sensory evaluations

Trinderup, Camilla H.; Møller, Flemming; Dahl, Anders Bjorholm; Conradsen, Knut

Published in:

Meat Science

Link to article, DOI:

10.1016/j.meatsci.2018.07.029

Publication date:

2018

Document Version

Peer reviewed version

Link back to DTU Orbit

Citation (APA):

Trinderup, C. H., Møller, F., Dahl, A. B., \& Conradsen, K. (2018). Investigation of pausing fermentation of salamis with multispectral imaging for optimal sensory evaluations. Meat Science, 146, 9-17.

https://doi.org/10.1016/j.meatsci.2018.07.029

\section{General rights}

Copyright and moral rights for the publications made accessible in the public portal are retained by the authors and/or other copyright owners and it is a condition of accessing publications that users recognise and abide by the legal requirements associated with these rights.

- Users may download and print one copy of any publication from the public portal for the purpose of private study or research.

- You may not further distribute the material or use it for any profit-making activity or commercial gain

- You may freely distribute the URL identifying the publication in the public portal 


\section{Accepted Manuscript}

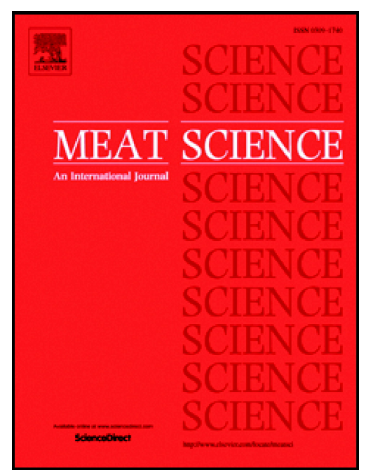

Camilla H. Trinderup, Flemming Møller, Anders Bjorholm Dahl, Knut Conradsen

PII:

S0309-1740(17)31527-9

DOI: doi:10.1016/j.meatsci.2018.07.029

Reference:

MESC 7643

To appear in:

Meat Science

Received date:

8 December 2017

Revised date:

19 July 2018

Accepted date:

23 July 2018

Please cite this article as: Camilla H. Trinderup, Flemming Møller, Anders Bjorholm Dahl, Knut Conradsen, Investigation of pausing fermentation of salamis with multispectral imaging for optimal sensory evaluations. Mesc (2018), doi:10.1016/j.meatsci.2018.07.029

This is a PDF file of an unedited manuscript that has been accepted for publication. As a service to our customers we are providing this early version of the manuscript. The manuscript will undergo copyediting, typesetting, and review of the resulting proof before it is published in its final form. Please note that during the production process errors may be discovered which could affect the content, and all legal disclaimers that apply to the journal pertain. 
Investigation of Pausing Fermentation of Salamis with Multispectral Imaging for Optimal Sensory Evaluations

Camilla H.Trinderup ${ }^{1,{ }^{*}}$ ctri@dtu.dk; Flemming Møller² flemming.moller@dupont.com; Anders Bjorholm Dahl ${ }^{1}$ abda@dtu.dk; Knut Conradsen ${ }^{1}$ knco@dtu.dk

${ }^{1}$ Technical University of Denmark, Richard Petersens Plads, 2800 Kgs. Lyngby, Denmark

${ }^{2}$ DuPont Nutrition Biosciences ApS, Edwin Rahrs Vej 38, 8220 Brabrand, Denmark

"Corresponding author address: Richard Petersens Plads, bldg. 324; 2800 Kgs. Lyngby; Denmark; Tel. $+4545253463$ 


\begin{abstract}
The fermentation process of salamis involves several parameters influencing taste, texture, and color of the salami. One significant parameter is the fermentation time. It is difficult to conduct sensory evaluations to assess the effect of time without introducing variation between observation days. It may be possible to overcome this by stalling or pausing the fermentation by deep-chilling the salamis. This study investigates the difference of non- and deep-chilled salamis with the use of a multispectral imaging system. The statistical investigation, based on image features relating to size, visual texture, and color of the sausages over time, showed that it may be possible to stall the fermentation process. It was shown that a statistical difference in the two kinds of samples is present. For the size feature the difference could be quantified into a number of days. However, for the important color feature only a statistical difference was observed, whereas the visual difference expressed in terms of $\Delta E_{a b}{ }^{*}$ was barely present.
\end{abstract}

Keyword Fermentation; salami; texture; multispectral imaging; color assessment 


\section{Introduction}

The visual changes during the fermentation process of salamis occur due to evaporation and acidification. The changes are expressed in terms of shrinkage of the salami diameter, a graduate color change and a texture change as visualized in Figure 1.

A sensory panel is a strong method to precisely determine quality parameters of food (Van Kleef et al., 2005), but variations in their assessment can occur, especially when the same product is assessed at different time points. This is of particular relevance for fermented products like salamis, where the sausage is developed over time. In order to avoid such variations it would be advantageous to present all fermentation stages of the salamis to the sensory panel at once. Having all fermentation stages could be achieved by starting the salami production at different time points, but this can cause variations between batches, which also should be avoided. Instead it would be beneficial to have a method for stopping or pausing the fermentation process, such that a sensory panel can be exposed to all fermentation stages at the same trial and perform an equal assessment of the salamis. This pausing of the fermentation process is possible by deep chilling the samples. It is however important that the deep-chilling does not influence the salami.

In this study we investigate how deep-chilling influence the appearance using multispectral images. To do this we provide a set of tools for processing and analyzing the multispectral images of salami. A vision system is an obvious choice for a comparison study like this, since the illumination is consistent across acquisition days, and therefore ensures equal and objective assessment properties across fermentation stages.

Image analysis is widely applied in assessment of food products. Several studies describe how computer vision systems and subsequent image analysis can be used in the process of describing various meat products (Valous et al., 2009; Chmiel et al., 2011). The studies relate to both color and visual texture of the products. Mendoza et al. (2006) reviewed how different vision systems were employed to assess color and other attributes of agricultural foods. Image analysis has also proven useful in the analysis of ripening stages for fruits and vegetables (Mendoza and Aguilera, 2004; Xing and De Baerdemaeker, 2005; Steinmetz et al., 1999), identification of previously frozen products (Brosnan and Sun, 2004; Sharifzadeh et al., 2013; Pu et al., 2015; Ropodi et al., 2018), and spoilage detection in meat (Dissing et al., 2012; Tsakanikas et al., 2016). Feng and Sun (2013) investigated Pseudomonas loads in chicken fillets using near infrared hyperspectral imaging. Based on multispectral imaging in the visible and near infrared (NIR) regions, Ma et al. (2014) presented a rapid and nondestructive method for determining the aerobic plate count (APC) in cooked pork sausages. Ropodi et al. (2017) used multispectral imaging in detection of minced beef adulteration with horse meat.

The feasibility of applying different data analytic methods in food science is addressed by several authors. Pu et al. (2015), were - among other things - investigating texture analysis, Ma et al. (2014) used partial least squares regression in calibrating relationships, Ropodi et al. 
(2018), and Tsakanikas et al. (2016) introduced methods related to machine learning. Work presented in this paper is inspired by the studies in Møller (2012).

We quantify appearance features related to size, color, and visual texture of fermented salami slices using image analysis. Our results showed subtle differences between deep-chilled and non-chilled samples. The differences were quantified by statistical models and principal component analysis (PCA). The analysis revealed a statistical difference, but this significance is not necessarily translatable to visual difference. Measured in the CIELAB color space this difference was below the human visible threshold.

\section{Materials and Methods}

During the fermentation process of a salami its circumference decrease and the color changes. Moreover, the firmness or texture of the product also changes. This time dependent change was captured by extracting descriptive features from multispectral images. These features were compared for the deep-chilled and non-chilled samples to investigate whether a time dependent change was present.

\subsection{Samples and Instrument}

The rate of the changes during the fermentation process is determined by factors like fermentation culture, smoke time, fat and meat content, $\mathrm{pH}$ drop, etc. In this study we considered four different recipes of starter cultures, and keept all other factors constant. This resulted in four different fermentation processes.

We studied samples from specific days (2, 3, 9, 14, 21 and 42) after fermentation start, as illustrated in Figure 1. The observation frequency in the beginning of the fermentation process is higher than in the end since the meat cures faster in this period (Huefner and Hertel, 2008). On these days, two samples of each recipe were taken from the smoker. From one sausage three slices of $\sim 2 \mathrm{~cm}$ were cut and imaged with the multispectral imaging system. The other sausage was placed in the cooler at $-2^{\circ} \mathrm{C}$ until day 42 , deep-chilling. At day 42 , these samples were taken out of the cooler and put in the refrigerator until they reached a temperature of $5^{\circ} \mathrm{C}$. Similar to the non-chilled samples, the deep-chilled samples were now sliced and imaged with the multispectral imaging system. Consequently, the chilled samples were stored at $-2^{\circ} \mathrm{C}$ for a different number of days when imaged.

This study has a total of $n=132$ observations. For the first five observation days, three replications of four recipes for both chilled and non-chilled samples were imaged. On the last observation day, only non-chilled samples were imaged, since the test series was finished and no more samples were deep-chilled.

In this study we employed the multispectral imaging system VideometerLab that depicts objects with a diameter of up to $10 \mathrm{~cm}$ using wavelength specific diffuse illumination, making it ideal for salami slices. The resulting images were $2056 \times 2056$ pixels with a pixel size of $45 \mu \mathrm{m}$. The instrument had 19 spectral bands -12 visible and 7 near-infrared bands. More specific information on the vision system can be found in Ljungqvist et al. (2014). 


\subsection{Image analysis}

Figure 2 presents the steps in the analysis of the multispectral image - from segmentation to feature extraction and statistical analysis. All steps will be described in the rest of this section.

\subsubsection{Segmentation}

Canonical discriminant analysis (CDA) was applied to segment the images. This supervised classification procedure use training areas of $k$ classes for defining a discriminator function, $\boldsymbol{Y}$ $=\boldsymbol{d}^{T} \boldsymbol{X} . \boldsymbol{d}$ is found by maximizing the Rayleigh coefficient

$$
R(\mathbf{d})=\frac{\mathbf{d}^{T} \mathbf{B d}}{\mathbf{d}^{T} \mathbf{W d}}
$$

corresponding to maximizing the ratio of the variation between $(\boldsymbol{B})$ relative to within classes $(\boldsymbol{W})$. We may obtain $k-1$ uncorrelated solutions with decreasing discriminative power.

We also applied normalized CDA (nCDA). In that case, the canonical discriminant score were normalized such that

$$
\mathbf{y}=\frac{\mathbf{y}-\overline{\mathbf{y}}}{\mathbf{s}_{y}}
$$

with $\overline{\mathbf{y}}$ and $\boldsymbol{s}_{\boldsymbol{y}}$ representing the estimated mean and standard deviation. This way we ensured canonical discriminant functions centered around zero, and hence an easy thresholding for segmentation was obtained.

The CDA and nCDA were used as segmentation tools. Though they are supervised methods they require minimum manual work for finding training areas. First a segmentation of background and salami was performed by CDA. The variation of the background is relatively small compared to the salami itself, and the method discriminates well. The next step was to identify pixels of meat and fat of the salamis. Here the training areas were found on day 9 samples, reflecting the variation present across all fermentation stages. The segmentation results were improved by performing a spectral pre-treatment. The pre-treatment is a pixelwise operation, where all bands were divided by the third spectral band $(435 \mathrm{~nm})$. The third band is left out of the subsequent nCDA, where an increase in Rayleigh coefficient was observed for the pretreated data. An example of the final segmentation is shown in Figure 3.

\subsection{Width of Salami}

From the segmentations of background and salami the width of the salami was found in the horizontal direction. In case the chilling process influenced the structure and evaporation properties of the salami it could lead to a difference in this physical parameter.

\subsection{Texture}


Figure 1 shows the fermentation progress for one recipe of salami, where a change in image texture over time is seen as an increase in blended meat and fat pixels. In this section references to texture will relate to the image texture given in terms of changes in pixel values. We will describe a possible way to capture the change in texture by considering gradient magnitude histograms (Lowe, 2004). The gradient of an image, $f$, is defined as

$$
\nabla f=\frac{\partial f}{\partial x} e_{x}+\frac{\partial f}{\partial y} e_{y}
$$

where $e_{x}$ and $e_{y}$ are the unit vectors in the $x$ - and $y$-directions respectively. The gradients in the image will be estimated by central differences (Elden et al, 2004). All the gradients in an image have an orientation, $\theta$, and a magnitude, $m$.

In this study we used the distribution of the gradient magnitudes of the salami images to characterize the fermentation process. The gradient magnitude histograms will be found at different scales, such that scale-dependent texture changes will be captured. The scales considered were found at five levels of a Gaussian pyramid. In each level of the pyramid, the image was convolved with a Gaussian kernel and the resolution is halved. The number of bins in the histogram was fixed for each scale. Moreover, the limits of the histogram were the same for all samples making the histograms comparable across samples. Figure 4 shows an example of gradient magnitude images at the five levels of the Gaussian pyramid. In this analysis we applied the background-salami mask found previously, such that the texture measure only represented the salami itself.

Several parameters influenced the histograms. The number of scales was chosen to be five in this case. The number of bins for each histogram was fixed at 64 . For each wavelength and number of bins, the histogram was found by concatenating the histograms for the five considered scales. An example of a concatenated histogram of a day 2 sample is seen in Figure 4.

After careful analysis it was decided to use the 17th band in the analys is of the changes in visual texture. This band gave good contrast between the meat and fat parts of the salami slices, and was therefore suitable for capturing the visual texture difference.

\subsection{Statistical Color Information}

From the rim of the salami, a gradual color change of the meat parts takes place during the fermentation process - see Figure 1. In order to capture the variation in meat color between the samples we defined a meat color scale based on an nCDA, using training areas from nonchilled samples at days 2 and 42. All samples were subsequently mapped to the obtained nCDA space. Negative values of the nCDA meat color scale reflect fresher meat, whereas the positive values represent the darker, fermented meat. It is important to mention that the nCDA was trained on the non-chilled samples, so the deep-chilling process is not already accounted for in the analysis of the color feature. The CDA loadings for each of the 19 spectral bands are summarized in Figure 5. In general the CDA measured the difference 
between the longer wavelengths, red $(645-660 \mathrm{~nm})$ and NIR $(890-970 \mathrm{~nm})$, which is consistent with a change in red meat color.

Results of using the statistical color scale are also seen in Figure 5. In order to highlight the spatial variation we extracted the average pixel value in annuli at predefined radii from the center of the salami. The maximum radius considered, $r_{\max }$, was defined as the average width across all samples. For the samples that had a maximum radius smaller than $r_{\max }$, the missing pixel averages were found by extrapolation. In the present study we used 31 equidistant radii. Thus for each salami sample an observation $\boldsymbol{Y}_{i}=\left(Y_{i, 1}, \ldots, Y_{i, 31}\right)^{T}, i=1, \ldots, 132$, was obtained, where $Y_{(i, j)}$ is the average nCDA colour value at radius $r_{j}$ for sample no $i$. The $Y$ coordinates are strongly serially correlated and in order to describe the spatial variation they were transformed into their principal components where the first two were retained for further analysis.

\subsubsection{CIELAB Color Information}

In the statistical color measurement, all spectral bands were employed to obtain a general measure of color change. This approach is well suited for identification of even subtle differences. In food research, it is common to measure color in the CIELAB color space, since this relates well to human perception of color (Larraín et al., 2008). We converted the pixel-wise multispectral information to $L^{*}, a^{*}$, and $b^{*}$ pixel values by a photometric imaging model (Trinderup et al., 2015). For the three color components we followed the same strategy as for the nCDA meat color scale and determined the average color value in annuli and subsequently analyzed the spatial variation by looking on the three sets of principal components of the CIELAB values $\left(Y_{i, 1}^{L}, \ldots, Y_{i, 31}^{L}\right)^{T},\left(Y_{i, 1}^{a}, \ldots, Y_{i, 31}^{a}\right)^{T}$, and $\left(Y_{i, 1}^{b}, \ldots, Y_{i, 31}^{b}\right)^{T}$.

Furthermore, we assessed the color differences between the deep-chilled and non-chilled samples by computing the $\Delta E_{a b}{ }^{*}$ value (see e.g. Trinderup et al. (2015)) for the annuli considered above, and also for the inner third of the salami which is commonly used for sensory panel scoring. The $\Delta E_{a b}{ }^{*}$ parameter measures color differences by the Euclidean distance in the CIELAB space.

\subsection{Statistical Analysis}

The statistical analyses of the features defined above were made using the procedures GLM, GLMSELECT and NLIN from SAS/STAT ${ }^{\circledR}$ software. As a first approximation we shall fit polynomial models like

$$
\begin{aligned}
& E\left(X_{i j}(t)\right)=\mu_{i j}(t)=\mu+c_{i}+r_{j}+\beta_{i j} t+\gamma_{i j} t^{2}, \\
& i \in\{0,1\}, \quad j \in\{1,2,3,4\}
\end{aligned}
$$

to the variable $X$. Here $t$ is a time point, $c$ and $r$ are the main effects of chilling and recipe; $i$ relates to deep-chilled/non-chilled and $j$ to recipe type. In order to identify simpler models we used stepwise model selection. Beal (2007) has compared different model selection criteria and found that Schwarz' Bayesian Information Criterion (SBC) gave superior results in a 
series of simulation studies. Using SBC we in most cases ended up with models not containing any recipe dependence, i.e.

$$
\mu_{1}(t)= \begin{cases}\alpha_{0}+\beta_{0} t+\gamma_{0} t^{2}, & i=0 \\ \alpha_{1}+\beta_{1} t+\gamma_{1} t^{2}, & i=1\end{cases}
$$

In many cases the second order $t$-term was common, i.e. we have

$$
\mu_{2}(t)= \begin{cases}\alpha_{0}+\beta_{0} t+\gamma t^{2}, & i=0 \\ \alpha_{1}+\beta_{1} t+\gamma t^{2}, & i=1\end{cases}
$$

The deep-chilling and subsequent thawing may release some water from cells and make it more available for evaporation, and these can be see an increased evaporation in the beginning, i.e. an accelerated thawing possibly corresponding to a time shift. Similarly other processes might accelerate due to the thawing proces, e.g. caused by the partial destruction of cells etc. Such accelerations can be accounted for by a model like:

$$
\mu_{3}(t)= \begin{cases}\alpha+\beta t+\gamma t^{2}, & i=0 \\ \alpha+\beta(t-\delta)+\gamma(t-\delta)^{2}, & i=1 .\end{cases}
$$

Here $\delta$ represents the time shift between deep-chilled and non-chilled samples. For the analysis of the width of the salami we used a model based on simple, physical assumptions about the evaporation from the salami. If we assume that the evaporation at time $t$ is proportional to the amount of water $y(t)$ in the salami, $y(t)$ will be exponentially decreasing. Therefore, it seems natural to expect that the volume and hence also the diameter will decrease asymptotically to a limit larger than zero (determined by the amount of dry matter). Taking the possible time shifts into consideration we ended up with the model

$$
\mu_{4}(t)= \begin{cases}\lambda+\exp \left(-\frac{t-\alpha}{\beta}\right), & i=0 \\ \lambda+\exp \left(-\frac{t-\alpha-\delta}{\beta}\right), & i=1 .\end{cases}
$$

The models in [5]- [8] were applied in the analys is of the features extracted from the multispectral images relating to width, texture and colour of the salamis.

\section{Results and Discussion}

\subsection{Width Features}

From the segmentations of background and salami the width of the salami was found in the horizontal direction. The width of the samples decreases after fermentation start, which can be seen in Figure 1. After day 21 it seemed like the shrinkage stalled. Figure 6 shows the development in width of salamis along with the fit of the three models [6], [7] and [8]. The residual standard deviations for the three models were $0.36,0.35$, and 0.47 , thus there were no substantial differences in the goodness of fit of the three models. 
In the fit of the models $\mu_{3}(t)$ and $\mu_{4}(t)$ the $\delta$ parameter was estimated to -0.505 and -0.977 respectively indicating that the deep-chilled samples were approximately 0.5 or 1 day ahead in the fermentation process compared to the non-chilled samples.

\subsection{Texture Analysis}

The forming and growth of ice crystals may cause leakage and thus lead to textural changes in frozen-thawed meat products. Pu et al. (2015) investigated texture analysis (histogram statistics on principal component images, gray level co-occurrence matrices, and gray levelgradient co-occurrence matrices) in the classification of frozen-then-thawed meat.

Since there is nothing canonical about the resolution of the multispectral images, we - as described in section 2.4 - decided to investigate texture features based on gradient magnitude at five different scales. With histograms of 64 bins, this resulted in a feature vector of length 320 for each sample.

We performed a PCA on this dataset consisting of 132 samples of the 320-dimensional texture vector. The first three principal components explained $80.7 \%$ of the variance in the histogram data, with the first PC explaining $\sim 64 \%$. As can be seen from the loadings given in Fig. 7, the first PC was dominated by the difference between the lower gradient magnitude values and the high gradient values at all scales. Therefore, a high value of PC1 corresponds to an image dominated by high gradients, i.e. sharp edges between fat and meat. A low value corresponds to few high gradients and many low, i.e. a more blurry image with more 'fuzzy' borders between fat and meat.

It is important to notice that we were not interested in discriminating between the deepchilled and non chilled samples. Had that been the case, e.g. Ropodi et al. (2018), and Tsakanikas et al. (2016) give good surveys of data-analytic tools for that, including support vector machines, partial least squares discriminant analysis, and Gaussian Mixture models. Our aim was to model a possible difference between non-chilled and deep-chilled samples. By a statistical analysis of the first PC we could to some extend establish whether the texture feature is descriptive of a potential difference between the non-chilled and chilled samples. Again we found a general linear model by a stepwise selection method from the model in [4]. The identified significant effects gave rise to a model as in [5] describing the time dependent texture change. The fit of this model to PC1 is seen in Fig. 7, where it is also obvious that the value of $\gamma_{1}$ is very close to zero. This difference in parameter for the second order polynomial term could be a consequence of missing data points for the chilled samples on day 42. In case we force the model to have the same polynomial term for the two types of treatment, we obtained a model as in [6]. The fit of this model is also shown in Figure 7.

The results indicate that there is a statistical difference in the texture features of the chilled and non-chilled samples corresponding to higher values of PC1 for the early deep-chilled samples. The early deep chilled samples have higher gradients between meat and fat and thus show a weaker effect of the fermentation. For the non-chilled samples we first see a decrease and then an increase on day 42 . The results for the first 3 weeks of fermentation indicate a 
decrease in the difference between deep-chilled and non-chilled samples. The increase in PC1 values for the non-chilled samples observed at day 42 might be due to the drying that takes place.

\subsection{Analysis of Statistical Color Scale}

The statistical meat color scale described in Section 2.5 was found as a feature for all samples. The CDA loadings for each of the 19 spectral bands are summarized in Figure 5. The CDA scores measures the difference between the longer wavelengths, red $(645-660 \mathrm{~nm})$ and NIR (890 - $970 \mathrm{~nm}$ ), which is consistent with a change in red meat color.

Fig. 8 shows the CDA color scale values for recipe 1 at the defined radii. Average values for all observation days are shown. The signals are similar for the remaining three recipes. The plot shows a general increase in nCDA value with time, more pronounced near the rim of the salami. This corresponds to a darkening of the red part of the meat, also seen clearly at the rim. Furthermore, the separation between the meat color for the non-chilled and deep-chilled samples becomes more dominant at the center of the salami on the later days of observation.

To explore the color co-variability of the salami samples we employed a PCA. The analysis showed that the first and second principal components (PC) explain 95.9\% and 3.6\% of the variance in the data respectively. The PC loadings in Figure 8 show that the first PC describes an average color across all radii. The second PC describes the contrast between the inner and outer part of the salami slices.

The model describing PC1 is similar to [5], and again a reparameterization is performed to reach a model as in [7], letting a time shift explain a substantial part of the difference between deep-chilled and non-chilled samples. The fits of both models are seen in Fig. 9. The same approach was employed to find a model for PC2. The resulting model is dependent on the chilling factor for all terms, and the fit is also shown in Fig. 9.

The residual standard deviations for the two models for PC1 are 1.36 and 2.23, i.e. within the same range so we may use the time-shifted model. The time shift parameter $\delta$ is estimated to -3.2 , so the average color for deep-chilled samples is approximately three days ahead of the non-chilled. For PC2, the residual standard deviations are 0.11 and 0.56 , i.e. five times larger for the time shifted model. In this case it is obviously not feasible to apply the latter model. This means that the contrast between the inner and outer part of the salamis are developing differently for deep-chilled and non-chilled samples although they show the same general trend with in increasing value for the first two weeks and then a decrease.

\subsection{CIELAB Color Analysis}

As described in Section 2.5.1 we also performed a statistical analysis of the CIELAB color components. We extracted the feature in the same manner as for the statistical meat color scale, and performed the same fitting to the models in [5] - [8]. This analysis showed that the individual color components, $L^{*}, a^{*}$, and $b^{*}$, were influenced by the chilling of the samples. In particular the $a^{*}$ component was influenced by the chilling, which relates well with the fact 
that it is the $a^{*}$ component that represent red color. But the statistical significant influence of the chilling may not represent a visual detectable difference.

As mentioned earlier, the inner third of the salamis is often used for color judgement by a sensory panel. Let $L_{i j}{ }^{*}(t)$ be the average $L^{*}$-value for the inner third of the three salami slices measured from recipe $j$ on day $t . i=0$ corresponds to non-chilled and $i=1$ to deep-chilled salamis. We assumed a two-way interaction model $E\left(L_{i j}{ }^{*}(t)\right)=\mu+c_{i}+r_{j}+d_{t}+\alpha_{i j}+\beta_{i t}+\gamma_{j t}$, where $\mu$ is the general level, $c_{i}, r_{j}$ and $d_{t}$ represented the effects of chilling, recipe, and day number (time), and the remaining parameters represent the two-way interaction terms. All parameters must satisfy the usual ANOVA constraints of summing to 0 . The variables (skipping the subscripts)

$$
\Delta L^{*}=L_{1 j}^{*}(t)-L_{0 j}^{*}(t), j \in\{1,2,3,4\}, t \in\{2,3,9,14,21\} . \quad[9]
$$

thus represent the differences between the chilled and the non-chilled samples in the $L^{*}$ color component. Similar expressions were obtained for the other two components. The observed values for recipe 1 are presented in Table 1 . The expected value of $\Delta L^{*}$ is $c_{1}-c_{0}+\alpha_{1 j}-\alpha_{0 j}+$ $\beta_{1 t}-\beta_{0 t}$. If there is no interaction between time and chilling, we get

$$
E\left(\Delta L^{*}\right)=E\left(L_{1 j}^{*}(t)-L_{0 j}^{*}(t)\right)=c_{1}-c_{0}+\alpha_{1 j}-\alpha_{0 j},
$$

i.e. for a fixed recipe, we have five random variables with the same mean value. We may therefore, using a $t$-test, investigate whether this mean can be assumed to be zero. Table 1 presents the $p$-values for these tests for recipe 1 samples.

The $p$-values showed a clear significance for the $\Delta a^{*}$ and $\Delta b^{*}$ values, and a border case for $\Delta L^{*}$. Thus we had a clear statistical difference between the color values. Table 1 also states the corresponding values of $\Delta E_{a b}{ }^{*}$. For all samples we got an average of approximately 2.1 and the maximum value is 2.9. The latter results give reason to believe that the color difference between chilled and non-chilled samples will be hard to detect for a sensory panel, since the human threshold is approximately 2.5 (Larraín et al., 2008). Thus, we see that the deep- and non-chilled samples have a statistical significant color difference, but it is most likely not visually detectable.

\section{Conclusion}

It should be emphasized that the purpose of the present paper was not to compare and evaluate different data analytic principles in the analysis of multispectral images of food samples, but to establish an analysis pipeline modeling the properties of interest for the food scientist. E.g. there are numerous methods for describing image texture, and possibly some might be as good (or even better) than the one described here. The virtues of the methods chosen are that they all give a description of such detail that it is possible to draw useful inferences.

Specifically, fermentation of deep-chilled and non-chilled salami was monitored by multispectral imaging. The basic features extracted from the multispectral images were 
width, color, and visual texture. These features showed that the chilled and non-chilled samples do differentiate from each other. The individual analyses of the features showed statistical differences. In the case of the width of the salami it was possible to quantify the difference in days of fermentation to something between a half and a full day. The color difference as measured in either the artificial CDA color or CIELAB values also showed statistical differences. However, quantifying the color difference by the $\Delta E_{a b}{ }^{*}$ showed a difference on the border of being visually detectable. A texture difference was also observed, but harder to relate to the sensory or physical parameter.

For the entire measurement period the differences between the CIELAB color values for the deep-chilled and non-chilled samples were below what is normally considered to be the detection limit for human visual assessments. As regards such assessments it is thus possible to stall the fermentation of salamis by deep-chilling so that different stages in the fermentation process may be assessed by a sensory panel at the same time point. The differences between deep-chilled and non-chilled samples could however be detected by suitable statistical analyses.

\section{Aknowledgement}

This work has been financed by the Center for Imaging Food Quality, a project funded by the Danish Council for Strategic Research (contract no 09-067039) within the Program Commission on Health, Food and Welfare. The experiments were conducted in collaboration with laboratory technicians at DuPont Nutrition Biosciences ApS. 


\section{References}

Beal, D. J. (2007). Information criteria methods in SAS for multiple linear regression models. SESUG Proceedings. Paper SA05. Raleigh, NC: North Carolina State University.

Brosnan, T. and Sun, D. (2004). Improving quality inspection of food products by computer vision - a review. Journal of Food Engineering, 61(1):3-16.

Chmiel, M., Slowinski, M., and Dasiewicz, K. (2011). Lightness of the color measured by computer image analysis as a factor for assessing the quality of pork meat. Meat science, 88(3):566-570.

Dissing, B. S., Papadopoulou, O. S., Tassou, C., Ersbøll, B. K., Carstensen, J. M., Panagou, E. Z., and Nychas, G.-J. (2012). Using Multispectral Imaging for Spoilage Detection of Pork Meat. Food and Bioprocess Technology, pages 1-12.

Elden, L., Wittmeyer-Koch, L., and Bruun Nielsen, H. (2004). Introduction to numerical computation - analysis and MATLAB illustrations. Studentlitteratur.

Feng, Y.-Z. and Sun, D.-W. (2013). Near-infrared hyperspectral imaging in tandem with partial least squares regression and genetic algorithm for non-destructive determination and visualization of pseudomonas loads in chicken fillets. Talanta, 109:74-83.

Huefner, E. and Hertel, C. (2008). Improvement of raw sausage fermentation by stressconditioning of the starter organism lactobacillus sakei. Current Microbiology, 57(5):490496.

Larraín, R. E., Schaefer, D. M., and Reed, J. D. (2008). Use of Digital Images to Estimate CIE Color Coordinates of Beef. Food Research International, 41(4):380-385.

Ljungqvist, M. G., Nielsen, O. H. A., Frosch, S., Nielsen, M. E., Clemmensen, L. H., and Ersbøll, B. K. (2014). Hyperspectral imaging based on diffused laser light for prediction of astaxanthin coating concentration. Machine Vision and Applications, 25(2):327-343.

Lowe, D. (2004). Distinctive Image Features from Scale-Invariant Keypoints. International Journal of Computer Vision, 60(2):91-110.

Ma, F., Yao, J., Xie, T., Liu, C., Chen, W., Chen, C., and Zheng, L. (2014). Multispectral imaging for rapid and non-destructive determination of aerobic plate count (apc) in cooked pork sausages. Food Research International, 62:902-908.

Mendoza, F. and Aguilera, J. M. (2004). Application of image analysis for classification of ripening bananas. Journal of food science, 69(9):E471-E477.

Mendoza, F., Dejmek, P., and Aguilera, J. (2006). Calibrated Color Measurements of Agricultural Foods Using Image Analysis. Postharvest Biology and Technology, 41(3):285295. 
Møller, F. (2012). Imaging Food Quality. IMM-PHD-2012. Technical University of Denmark.

Pu, H., Sun, D.-W., Ma, J., and Cheng, J.-H. (2015). Classification of fresh and frozenthawed pork muscles using visible and near infrared hyperspectral imaging and textural analysis. Meat Science, 99:81-88.

Ropodi, A. I., Panagou, E. Z., and Nychas, G.-J. E. (2017). Multispectral imaging (msi): A promising method for the detection of minced beef adulteration with horsemeat. Food Control, 73:57-63.

Ropodi, A. I., Panagou, E. Z., and Nychas, G.-J. E. (2018). Rapid detection of frozen-thenthawed minced beef using multispectral imaging and fourier transform infrared spectroscopy. Meat Science, 135:142-147.

Sharifzadeh, S., Clemmensen, L. H., Løje, H., and Ersbøll, B. K. (2013). Statistical Quality Assessment of Pre-fried Carrots Using Multispectral Imaging, pages 620-629. Lecture Notes in Computer Science. Springer.

Steinmetz, V., Roger, J. M., Moltó, E., and Blasco, J. (1999). On-line Fusion of Colour Camera and Spectrophotometer for Sugar Content Prediction of Apples. Journal of Agricultural Engineering Research, 73(2):207-216.

Trinderup, C. H., Dahl, A., Jensen, K., Carstensen, J. M., and Conradsen, K. (2015). Comparison of a multispectral vision system and a colorimeter for the assessment of meat color. Meat Science, 102(0):1-7.

Tsakanikas, P., Pavlidis, D., Panagou, E., and Nychas, G.-J. (2016). Exploiting multispectral imaging for non-invasive contamination assessment and mapping of meat samples. Talanta, 161:606-614.

Valous, N. A., Mendoza, F., Sun, D.-W., and Allen, P. (2009). Colour calibration of a laboratory computer vision system for quality evaluation of pre-sliced hams. Meat science, 81(1):132-141.

Van Kleef, E., van Trijp, H. C. M., and Luning, P. (2005). Consumer research in the early stages of new product development: A critical review of methods and techniques. Food Quality and Preference, 16(3):181-201.

Xing, J. and De Baerdemaeker, J. (2005). Bruise detection on 'Jonagold' apples using hyperspectral imaging. Postharvest Biology and Technology, 37(2):152-162. 
Table $1 L^{*}, a^{*}$, and $b^{*}$ differences for the inner third of recipe 1 samples. The $t$-test values relate to a paired $t$-test of the differences for each of the color components. Finally, the corresponding $\Delta E_{a b}{ }^{*}$ values for the observation days are also expressed.

\begin{tabular}{|l|c|c|c|c|c|c|}
\hline & Day 2 & Day 3 & Day 9 & Day 14 & Day 21 & $p$ \\
\hline$\Delta L^{*}$ & -0.1240 & 0.7306 & 1.2825 & 1.4279 & 2.4140 & 0.0517 \\
\hline$\Delta a^{*}$ & -1.0667 & -1.173 & -1.5639 & -1.5332 & -0.7242 & 0.0016 \\
\hline$\Delta b^{*}$ & -0.8394 & -0.9134 & -1.8387 & -2.0282 & -0.9888 & 0.0064 \\
\hline$\Delta E_{a b}^{*}$ & 1.3630 & 1.6176 & 2.7333 & 2.9160 & 2.7073 & \\
\hline
\end{tabular}


Figure 1 Fermentation process for one recipe of salami at day 2, 3, 9, 14, 21, and 42 after production (ordered from upper left to lower right). These images are obtained from multispectral images as false color composite based on three spectral bands $(660 \mathrm{~nm}, 470 \mathrm{~nm}$, $435 \mathrm{~nm})$.

Figure 2 Flow diagram of the feature extraction from the multispectral images.

Figure 3 Pseudo RGB image of a sample from day 14 with background and meat and fat segmentations.

Figure 4 Gradient magnitude images at the five levels of the Gaussian Pyramid and the resulting concatenated histogram.

Figure 5 From left right: CDA loadings for the statistical meat color scale. Each loading refers to a spectral band. nCDA meat color scale. The darker blue is fresh meat, whereas yellow and orange represent darker red, fermented meat.

Figure 6 Width feature with non-linear fits. From left to right: Fit of polynomial, time-shifted parabolas, and exponential function.

Figure 7 Left: PC loadings for the first three principal components of the concatenated histogram of texture features. Middle and right: $\mathrm{PC} 1$ and corresponding fits.

Figure 8 Left: Color distribution through a salami of recipe 1 as expressed in terms of the statistical meat color scale found by CDA. The value increases with fermentation time. Middle: Correlation between $r_{1}$ and $r_{2}, \ldots, r_{31}$. Right: PC loadings.

Figure 9 Top: Fit to PC1 and PC2 of statistical color scale. Bottom: Modified fit to PC1 and PC2 - two parabolas with a time shift. 

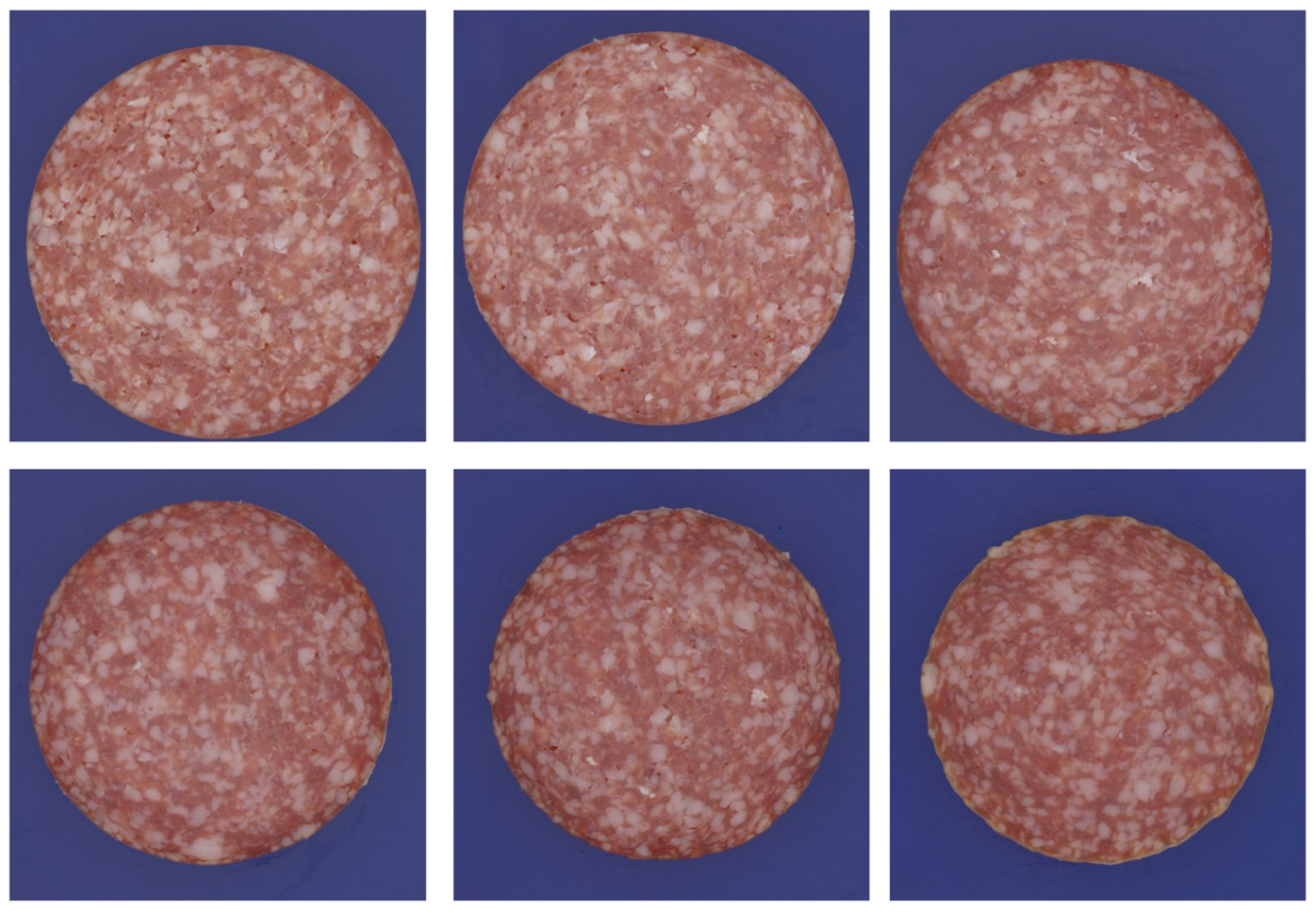

Figure 1 


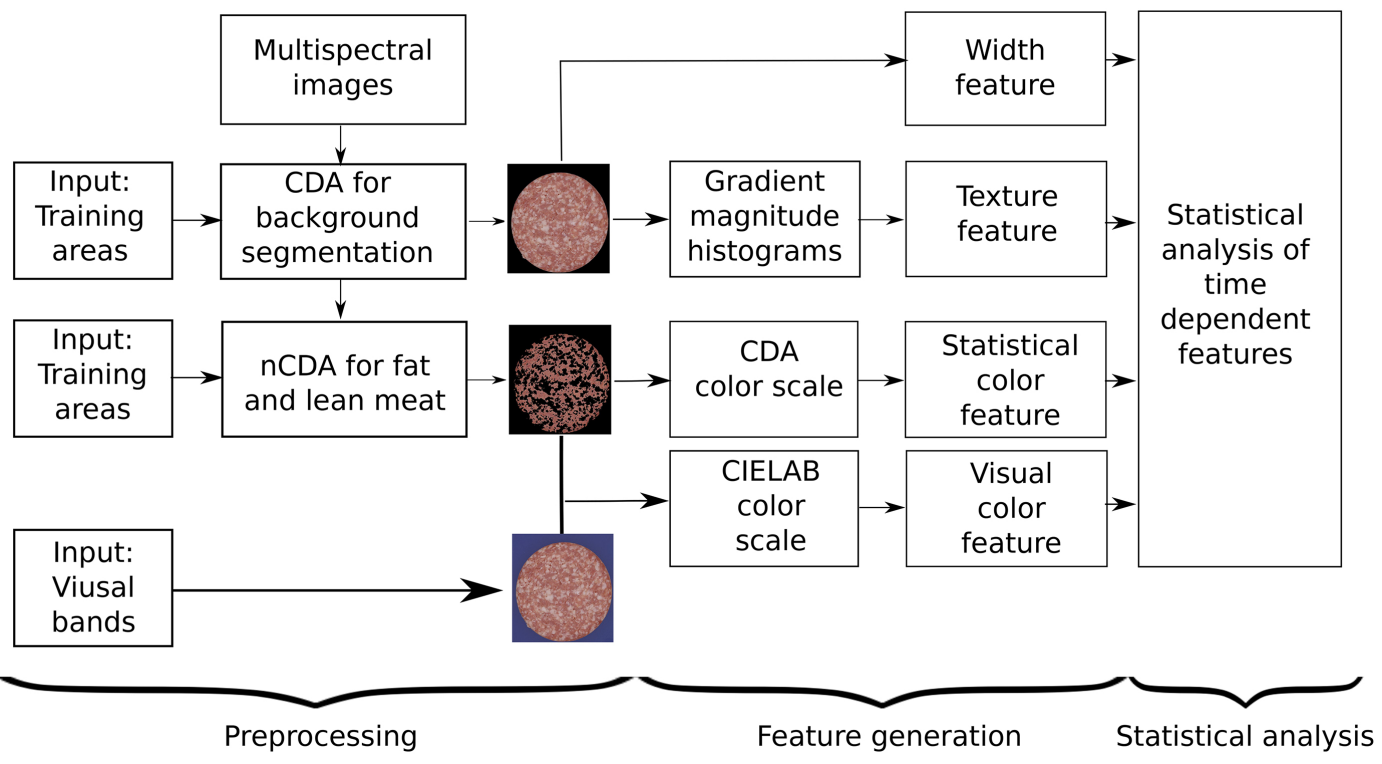

Figure 2 

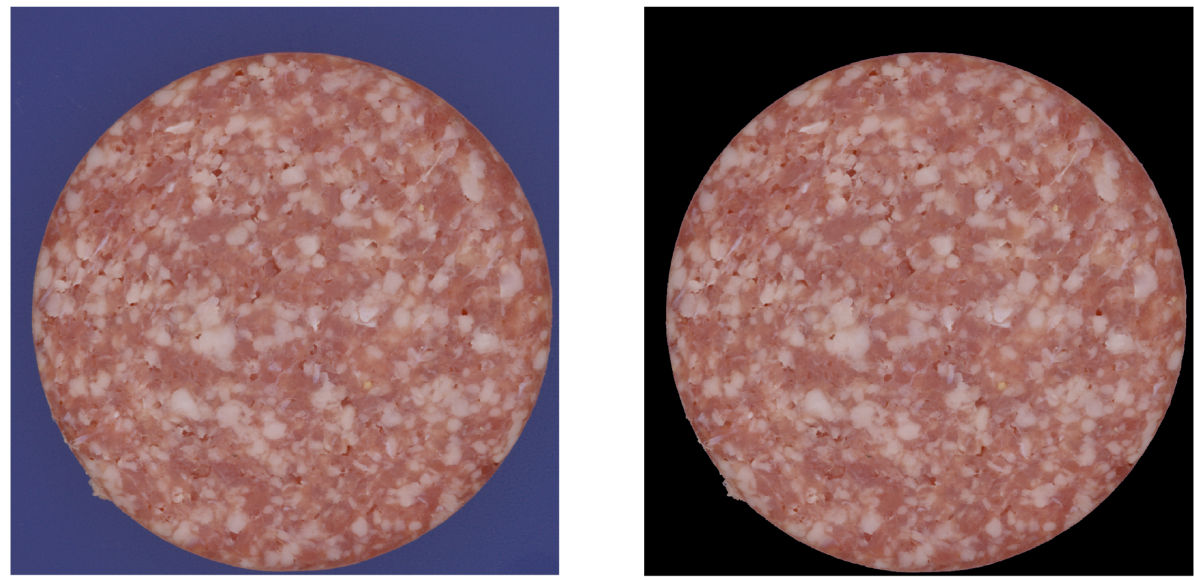

\section{$1+2$}

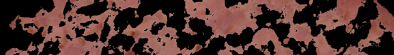
(8); ;

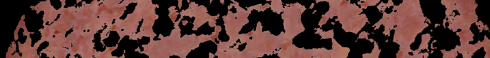

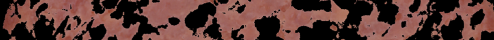

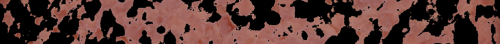
3.

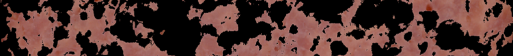

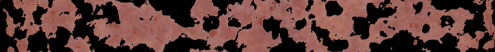

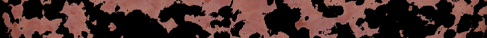

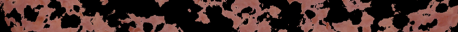

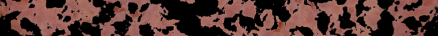

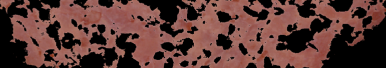$$
x-6,20+5=0
$$

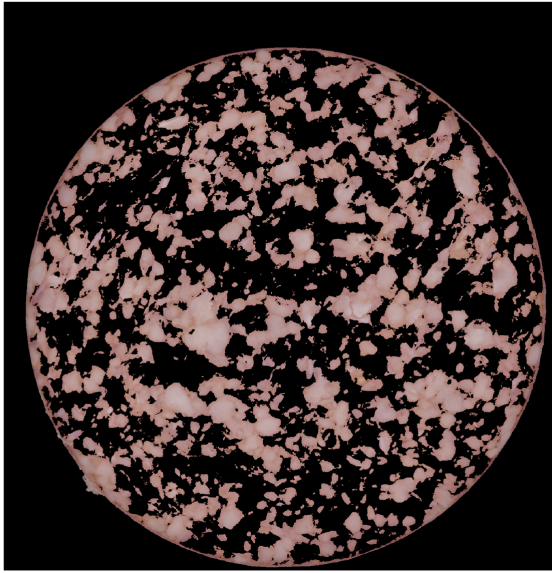

Figure 3 

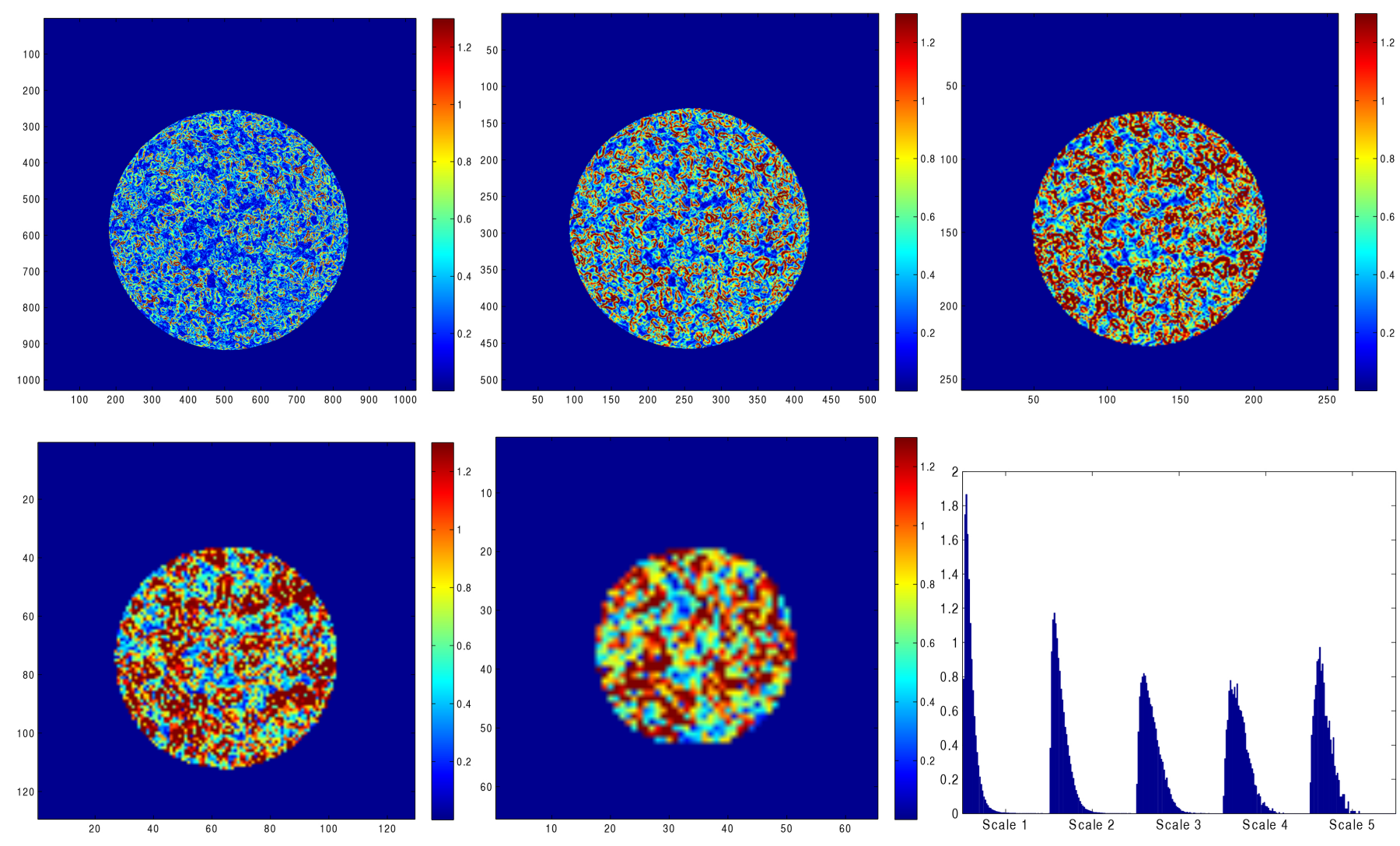

Figure 4 

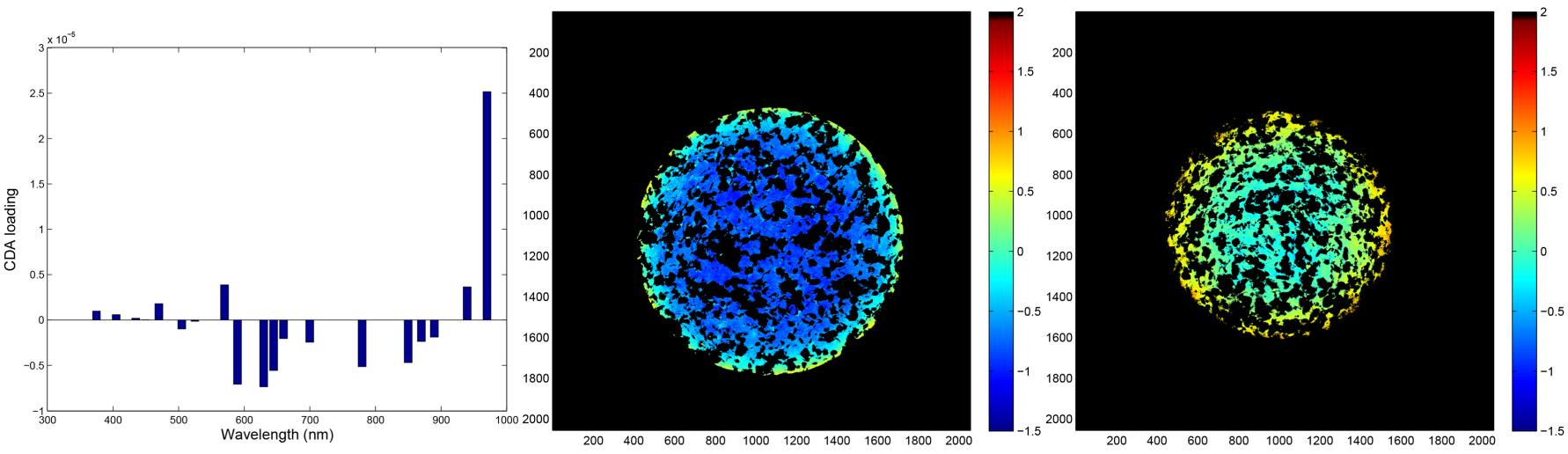

Figure 5 

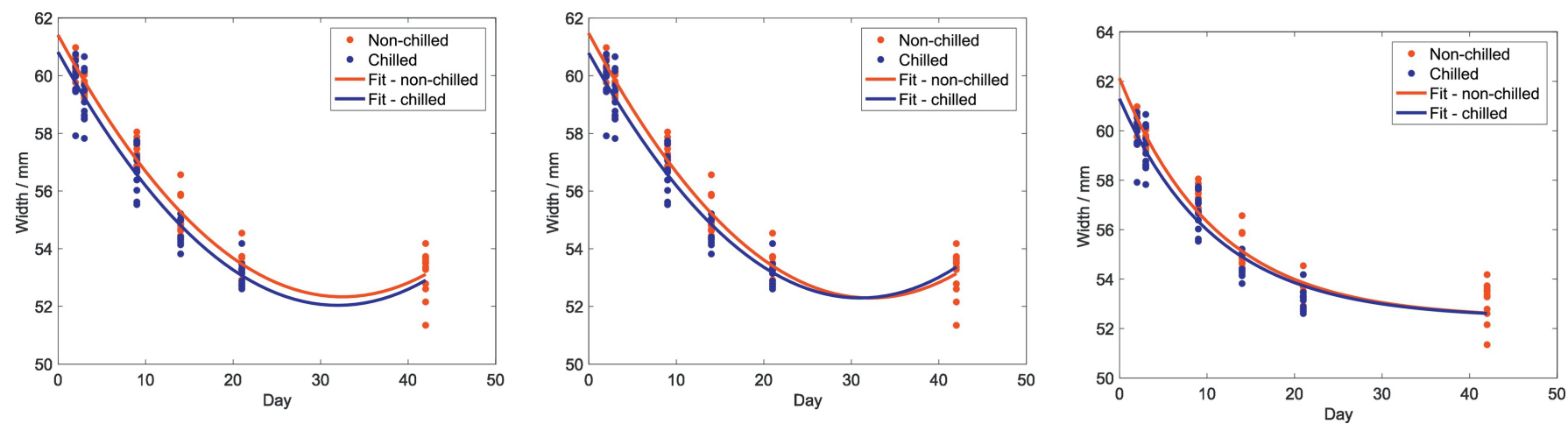

Figure 6 
CDA values for Recipe 1

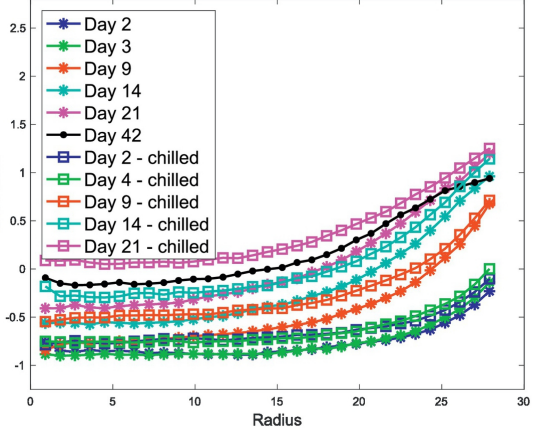

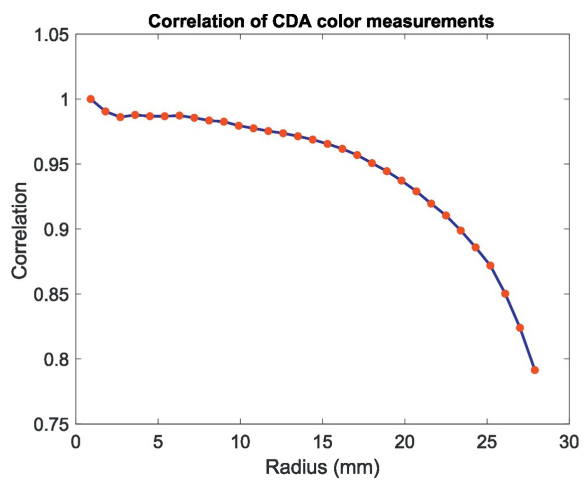

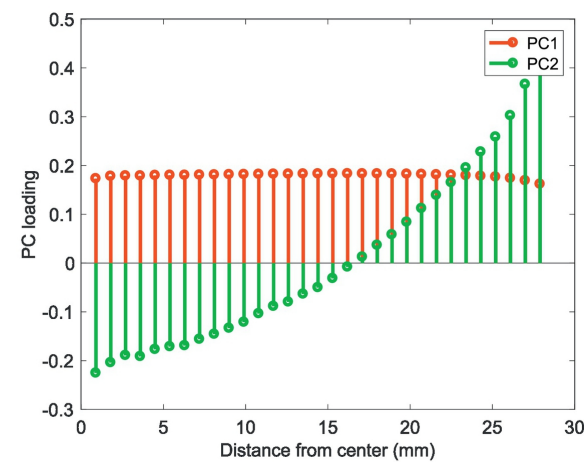

Figure 8 

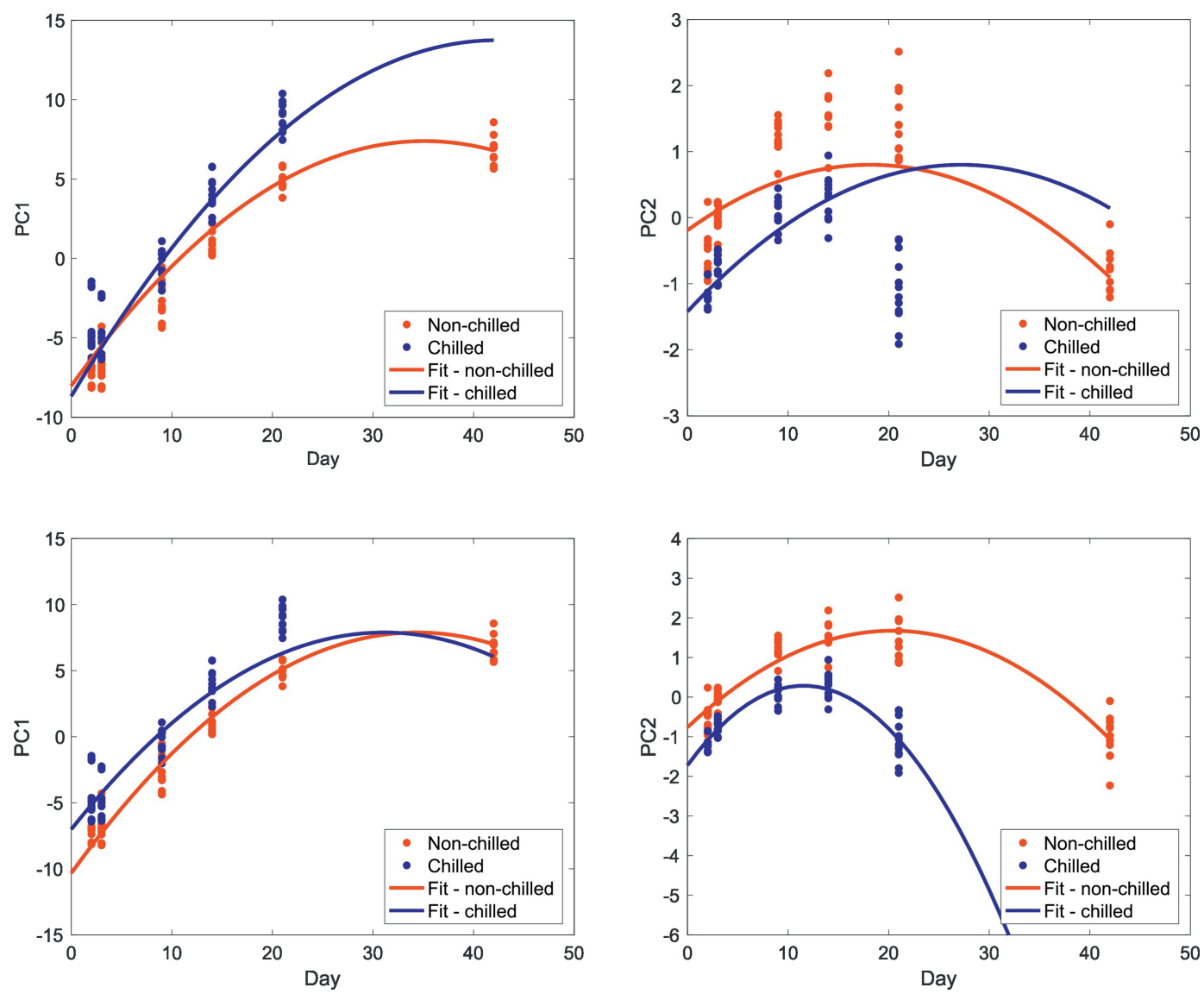

Figure 9 